\title{
Experimental and numerical study of buoyancy-driven single bubble dynamics in a vertical Hele-Shaw cell
}

Xue Wang, Bart Klaasen, Jan Degrève, Bart Blanpain, and Frederik Verhaeghe

Citation: Physics of Fluids 26, 123303 (2014);

View online: https://doi.org/10.1063/1.4903488

View Table of Contents: http://aip.scitation.org/toc/phf/26/12

Published by the American Institute of Physics

\section{Articles you may be interested in}

Volume-of-fluid simulations of bubble dynamics in a vertical Hele-Shaw cell

Physics of Fluids 28, 053304 (2016); 10.1063/1.4948931

Retarded motion of bubbles in Hele-Shaw cells

Physics of Fluids 8, 3229 (1998); 10.1063/1.869113

Bubble motion in a Hele-Shaw cell

The Physics of Fluids 31, 18 (1998); 10.1063/1.866566

The effect of surface tension on the shape of a Hele-Shaw cell bubble

The Physics of Fluids 29, 3537 (1998); 10.1063/1.865831

Droplet migration in a Hele-Shaw cell: Effect of the lubrication film on the droplet dynamics

Physics of Fluids 28, 062001 (2016); 10.1063/1.4952398

Scaling law for bubbles rising near vertical walls

Physics of Fluids 28, 062101 (2016); 10.1063/1.4948464

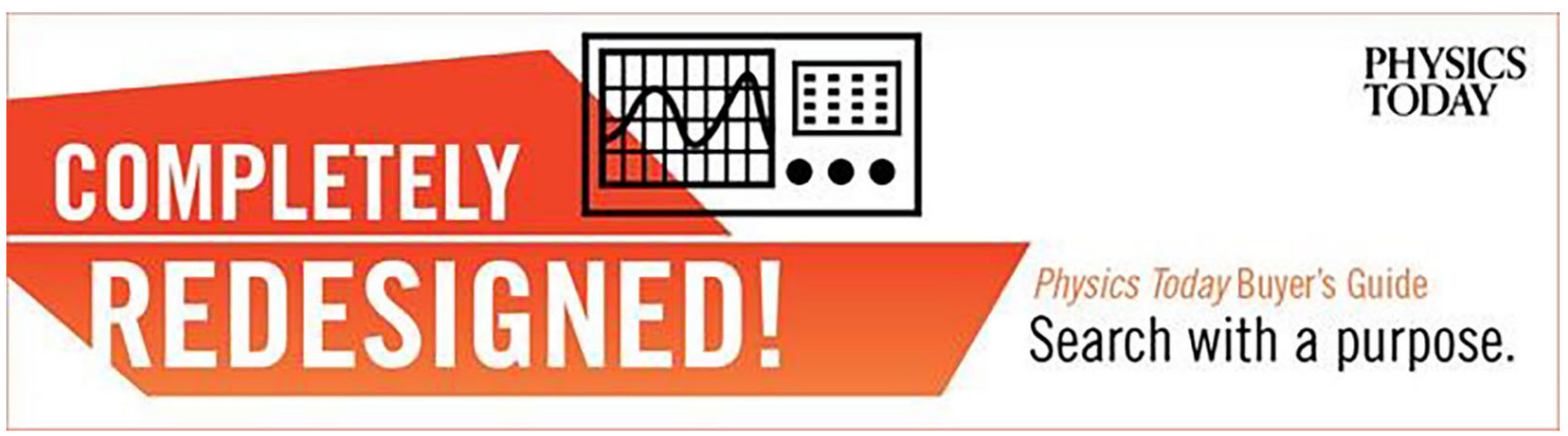




\title{
Experimental and numerical study of buoyancy-driven single bubble dynamics in a vertical Hele-Shaw cell
}

\author{
Xue Wang, ${ }^{1, a)}$ Bart Klaasen, ${ }^{2}$ Jan Degrève, ${ }^{3}$ Bart Blanpain, ${ }^{1}$ and \\ Frederik Verhaeghe ${ }^{1,2}$ \\ ${ }^{1}$ Department of Metallurgy and Materials Engineering, KU Leuven, Kasteelpark Arenberg \\ 44, bus 2450, 3001 Leuven, Belgium \\ ${ }^{2}$ Umicore Group Research and Development, Watertorenstraat 33, 2250 Olen, Belgium \\ ${ }^{3}$ Department of Chemical Engineering, KU Leuven, Willem de Croylaan 46, bus 2423, \\ 3001 Leuven, Belgium
}

(Received 18 March 2014; accepted 4 November 2014; published online 12 December 2014)

\begin{abstract}
Buoyancy-driven single bubble behaviour in a vertical Hele-Shaw cell with various gap Reynolds numbers $\operatorname{Re}(h / d)^{2}$ has been studied. Two gap thicknesses, $h=0.5 \mathrm{~mm}$ $\left(\operatorname{Re}(h / d)^{2}=1.0-8.5\right)$ and $1 \mathrm{~mm}\left(\operatorname{Re}(h / d)^{2}=6.0-50\right)$ were used to represent low and high gap Reynolds number flow. Periodic shape oscillation and path vibration were observed once the gap Reynolds number exceeds the critical value of 8.5. The bubble behaviour was also numerically simulated by taking a two-dimensional volume of fluid method coupled with a continuum surface force model and a wall friction model in the commercial computational fluid dynamics package Fluent. By adjusting the viscous resistance values, the bubble dynamics in the two gap thicknesses can be simulated. For the main flow properties including shape, path, terminal velocity, horizontal vibration, and shape oscillation, good agreement is obtained between experiment and simulation. The estimated terminal velocity is $10 \%-50 \%$ higher than the observed one when the bubble diameter $d \leq 5 \mathrm{~mm}, h=0.5 \mathrm{~mm}$ and $9 \%$ higher when $d \leq 18 \mathrm{~mm}, h=1.0 \mathrm{~mm}$. The estimated oscillation frequency is $50 \%$ higher than the observed value. Three-dimensional effects and spurious vortices are most likely the reason for this inaccuracy. The simulation confirms that the thin liquid films between gas bubbles and the cell walls have a limited effect on the bubble dynamics. (C) 2014 AIP Publishing LLC. [http://dx.doi.org/10.1063/1.4903488]
\end{abstract}

\section{INTRODUCTION}

A Hele-Shaw cell confines a bubble in a narrow gap between two flat parallel plates. ${ }^{1}$ The thickness of the gap between the plates $(h)$ is typically very small (in the order of $1 \mathrm{~mm}$ ). When the confinement ratio $h / d<<1$ ( $d$, the equivalent diameter of the bubble), the bubble inside the cell is flattened and loses the freedom in the third dimension, hence the bubble flow can be treated as two-dimensional. ${ }^{2}$ Apart from the confinement ratio, the bubble motion in the Hele-Shaw cell is determined by the complex interplay of viscous forces, inertial forces, and surface tension. Depending on the relative importance of these forces, the diversity of bubble dynamics can be represented. When the bubble Reynolds number is very small, i.e., $\operatorname{Re}<<1(\operatorname{Re}=\rho u d / \mu, \rho$ is the mass density of the fluid, $u$ the velocity of the bubble, and $\mu$ the dynamic viscosity of the fluid), the inertial forces are negligible, only viscous forces and surface tension are important. This regime is applicable for gas bubbles in a horizontal Hele-Shaw cell involving a highly viscous liquid, which has been studied analytically and experimentally. ${ }^{1-6}$ When the bubble Reynolds number is moderate or high, i.e., $R e \geq 1$, the inertial forces cannot be neglected, and all three forces are significant. The moderate $R e$ regime ( $R e$ in the order of 100) refers to a gas bubble in a tilted Hele-Shaw cell filled with a low viscous

\footnotetext{
a) Author to whom correspondence should be addressed. Electronic mail: xwang82@gmail.com
} 
liquid, while the high $R e$ regime ( $R e$ in the order of 1000) corresponds to the bubble behaviour in a vertical Hele-Shaw cell. The governing non-dimensional numbers for an unbounded two-dimensional buoyancy-driven bubble in the Hele-Shaw cell include the Archimedes number $(A r=\rho \sqrt{g d} d / \mu)$, $h / d$ and the Bond or Eötvös number $\left(B o=E o=\rho g d^{2} / \sigma\right), \sigma$ is the surface tension, and $g$ is the gravitational acceleration. When the cell width $W$ is finite and the bubble is large, $h / W$ becomes influential and it can replace $h / d$ when $r_{c} / W>0.7$, where the dimension control changes from bubble dimension to cell width dimension and the bubble becomes a gas $\operatorname{slug}^{7}\left(r_{c}\right.$, the radius of curvature at the front stagnation point). In addition, the gap Reynolds number $\operatorname{Re}(h / d)^{2}$ is also an important parameter, which compares the in-plane inertial time scale and the in-gap viscous time scale. ${ }^{8}{ }^{8} \mathrm{It}$ is dependent on both $A r$ and $h / d$. Kelley and $\mathrm{Wu}^{10}$ studied the path instability of a circular bubble at moderate $R e$ and concluded that a critical Reynolds number $(\approx 200)$ controls the path instability. Bush $^{11}$ reported a peculiar wake structure of a penny-shaped air bubble at moderate $R e$. Using the same experimental technique, Bush and Eames ${ }^{12}$ examined the fluid displacement associated with the rectilinear motion of oblate ellipsoidal/elliptical-cap bubbles. For these studies, only constant bubble shapes in a tilted Hele-Shaw cell were observed. Kawaguchi's group ${ }^{13-15}$ and Roig et al. ${ }^{16}$ expanded the studies to a large range of bubble sizes in a vertical cell at high Re. Kawaguchi et al ${ }^{13}$ examined the steady-state bubble shape, trajectory, and peripheral length in water as a function of $E o$ (Eo $\leq 40$, $\left.A r \leq 7 \times 10^{3}, h=1.0 \mathrm{~mm}, W=5 \mathrm{~cm}\right)$ and pointed out that $E o$ is the controlling parameter of the path instability. This work was further extended to polymer solutions ${ }^{14}$ and to transient states. ${ }^{15}$ Roig et al. ${ }^{16}$ investigated the path and shape oscillation of bubbles in water in a wider $R e$ range $(E o \leq 140$, $\left.A r \leq 15 \times 10^{3}, h=1.0 \mathrm{~mm}, W=40 \mathrm{~cm}\right)$. Although the cell width values in their experiments are different, since an identical gap thickness was used, their results are consistent, i.e., the shape oscillation and path instability are coupled in most of the $A r$ range $\left(600 \leq A r \leq 4 \times 10^{3}\right.$ in Ref. 13 and $100 \leq A r \leq 6 \times 10^{3}$ in Ref. 16). In all these experiments, the governing parameter $h / d$ was adjusted only by changing the initial bubble size while the cell gap thickness was fixed. As far as it is known, the effect of gap thickness on the bubble dynamics in a vertical Hele-Shaw has not been studied in detail and the path and shape responses on gap thickness variations remain unknown.

In contrast to the availability of the experimental results on diverse high-Reynolds-number bubble shapes and paths, only the large spherical-cap bubbles were analytically studied. ${ }^{7}$ The theoretical terminal velocity of the bubbles is given as $u=\varphi \sqrt{g d}$ (with $\varphi$, a constant in a given geometry) and Roig et al. ${ }^{16}$ fitted their experimental data with $\varphi=0.42(R e>3000)$. In this equation, the velocity is assumed to be related to the bubble size only, and therefore, the effect of the gap thickness $h$ on the terminal velocity is not incorporated and unknown. To understand the bubble dynamics in a more fundamental way, a numerical simulation by taking all related forces into account has to be conducted. Although the numerical method has been widely used to study the formation and dynamics of a single bubble in other media ${ }^{17}$ and other types of flows in the Hele-Shaw cell, ${ }^{18-21}$ direct simulation of high-Reynolds-number bubble flow in the vertical Hele-Shaw cell filled with water has not been found in the open literature. In the present study, a 2D volume of fluid (VOF) method will be used to simulate the bubble dynamics in the vertical Hele-Shaw cell. The same method was used to simulate a free rising bubble. ${ }^{22,23}$ Different from these studies, Darcy's law is added in the model to describe the in-gap wall friction. By doing this, the Hele-Shaw cell high-Reynolds-number bubble dynamics can be simulated quantitatively and the effect of gap thickness can be incorporated easily and accurately.

\section{EXPERIMENTAL SETUP}

A vertical Hele-Shaw cell with two gap thicknesses has been constructed in the experiment: (1) $h=0.5 \mathrm{~mm}( \pm 0.02 \mathrm{~mm})$, for low gap Reynolds number bubble behaviour; (2) $h=1 \mathrm{~mm}( \pm 0.02 \mathrm{~mm})$, for high gap Reynolds number bubble behaviour. The other two dimensions of the cells were identical, i.e., $50 \mathrm{~mm}$ in width and $500 \mathrm{~mm}$ in height. The equipment is as described in Ref. 24 . The cell was made of soda-lime glass with a thickness of $10 \mathrm{~mm}$. The glass plates were washed in advance with Piranha solution ( $3: 1$ mixture of $96 \% \mathrm{H}_{2} \mathrm{SO}_{4}$ and $30 \% \mathrm{H}_{2} \mathrm{O}_{2}$ ) to remove organic residues and hydroxylate surfaces, making the plates highly hydrophilic. A bubble was generated with a syringe needle (inner diameter range $0.3-0.8 \mathrm{~mm}$ ) controlled by an electromagnetic micro valve (brand is Burker). 
By varying the time step of the valve and the needle inner diameter, the initial bubble size could be controlled. Technically pure nitrogen gas $(99.9 \%)$ and ultrapure water $(18.2 \mathrm{M} \Omega \mathrm{cm}$ resistivity at $25^{\circ} \mathrm{C}$ produced by Millipore Direct-Q 3 UV ultrapure water system) were used to avoid the effect of surface active agents. Thin liquid films were formed between the gas bubble and the wall due to the total wetting of water on the washed glass cell. The images of the bubble were recorded with a Tesin Cyclocam high speed video camera at a frame rate of $250 \mathrm{fps}$ (resolution $640 \times 480$ pixels). The shutter time in the experiment was $2 \mathrm{~ms}$. The recorded image frames were sequentially analysed with a Matlab-based processing script. In each frame, the area $S$, perimeter $L$, and gravity centre $(x, y)$ of the bubble were determined by mapping the bubble contour. The equivalent diameter $d$ of the two-dimensional bubble was calculated as $d=2(S / \pi)^{0.5}$; the terminal velocity $V_{y}$ as $V_{y}=\Delta y / \Delta t$ and the horizontal velocity $V_{x}$ as $V_{x}=\Delta x / \Delta t$, where $\Delta t$ is the time step.

\section{NUMERICAL SIMULATION}

Figure 1 schematically illustrates the buoyancy-driven single bubble flow in the vertical HeleShaw cell. As mentioned previously, the bubble motion in the Hele-Shaw cell (when $h / d<<1$ ) can be treated as two-dimensional. Roig et al. ${ }^{16}$ confirmed that the motion is less sensitive to threedimensional effects when $h / d \leq 0.5$. However, the viscous drag in the third dimension needs to be considered and coupled properly in the simulation. In this paper, a gap-averaged 2D simulation is used, in which assumptions are made according to the flow properties in the third dimension: (1) the effects of the thin films due to total wetting are neglected (Figure 1(b)); (2) the third-dimensional velocity distribution can be averaged (Figure 1(d)).

As for assumption (1), there are two equations that can be used to calculate the liquid film thickness $\left(2 \delta_{f i m}=1.337 h(\mathrm{Ca})^{2 / 3}\right.$ in Ref. 4 and $2 \delta_{f i m}=0.66 h(\mathrm{Ca})^{2 / 3} /\left(1+3.33(\mathrm{Ca})^{2 / 3}\right)$ in Ref. 25 , $C a=\mu u / \sigma)$. These yield a similar result, i.e., $2 \delta_{f i m}=\alpha h(\alpha=0.06-0.08)$, which is quite small. Meanwhile, Roig et al. ${ }^{16}$ studied the drag force due to the liquid films by using a scaling law and pointed out that the drag force depends on the boundary conditions at the bubble and thin film interface: if the interface is free of contaminants, a zero-shear-stress boundary condition applies and no extra force is exerted by the thin film; the contaminated interface becomes rigid and a no-slip boundary condition applies; while a partially contaminated interface may have a non-zero viscous shear stress. In their experiment, distilled water was used and it was found that the drag due to the thin films is negligible. In our experiment, ultrapure water was used and the glass plates were washed with Piranha solution, so the effects of contamination can be neglected and the drag force due to total wetting is assumed to be zero.

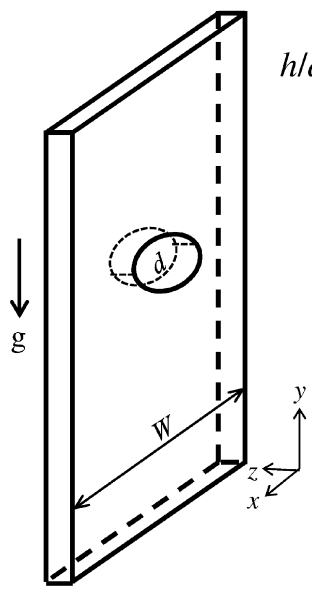

(a)

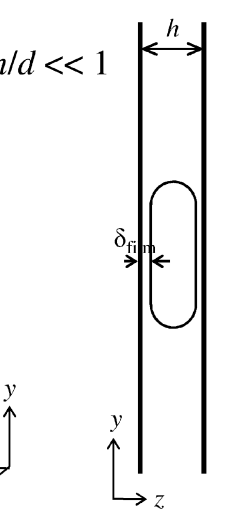

(b)

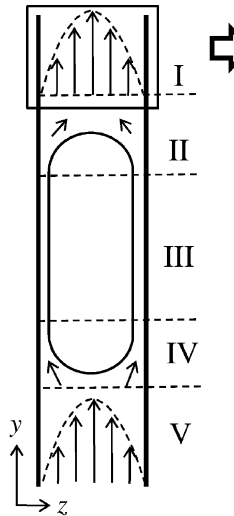

(c)
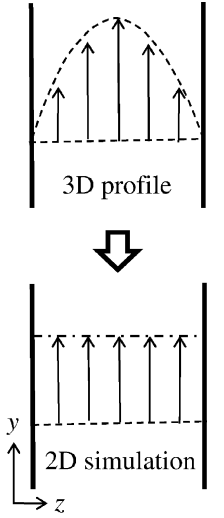

(d)

FIG. 1. Illustration of the Hele-Shaw cell. (a) Schematic view of the three-dimensional cell. (b) Side view of a bubble rising within the gap. (c) The velocity vectors within the gap. (d) Simplification of velocity profile in 2D simulation. 
As for the assumption (2), Beavers $e t$ al. ${ }^{26}$ concluded that transition to turbulent flow only occurs when the cell Reynolds number $R e=\rho u h / \mu$ (the characteristic length is gap thickness $h$ ) exceeds 2200 , so the laminar flow can be preserved safely in all cases once the velocity remains below 0.2 $\mathrm{m} / \mathrm{s}$ and the cell thickness is below $9 \mathrm{~mm}$ simultaneously. When the flow is far away from the bubble interface, the velocity distribution is parabolic due to Poiseuille flow (regions I and V in Figure 1(c)). The velocity distribution in the third dimension can be averaged in our $2 \mathrm{D}$ simulation by adding an averaged additional force in the momentum equation, which is illustrated in Figure 1(d). Inside the bubble (region III in Figure 1(c)), the velocity distribution depends on the thin films since the thin films can change the boundary conditions at the bubble-liquid film interface. However, their effects are negligible in our case and Poiseuille flow is assumed. Near the curved bubble interface (regions II and IV in Figure 1(c)), the flow streamline is bent and confined by the surface tension in the third dimension, the viscous dissipation is therefore increased. ${ }^{27}$ The three-dimensional effects are difficult to quantify and only qualitative estimations can be made here: large gap thickness and small bubble size lead to high three-dimensional effects. In the simulated gap thickness range ( $h=0.5-1 \mathrm{~mm})$ and bubble size range $(d=3-25 \mathrm{~mm})$, we assume these effects are negligible and the flow in the gap follows the Poiseuille flow.

Other assumptions include: the fluids are incompressible, the entire system is isothermal so that constant fluid properties for both phases are guaranteed, and surface tension $\sigma$ is constant.

The main three simulation methods available for gas-liquid multiphase flow with sharp interface include the front tracking method, the level-set method, and the VOF method. ${ }^{28}$ The VOF method is used in the present study due to its simplicity, robustness, and intrinsic mass conservation. ${ }^{29}$

\section{A. Governing equations}

Based on the above assumptions, the bubble dynamics in a vertical Hele-Shaw cell can be numerically simulated by a gap-averaged two-dimensional multiphase model. In the VOF method, a single set of momentum and continuity equations for unsteady, incompressible, immiscible two-phase flow is applied to both phases. The tracking of the interfaces between the phases is accomplished by the solution of the continuity equation for the volume fraction of each fluid through the domain:

continuity

$$
\frac{\partial \rho}{\partial t}+\nabla \cdot(\rho \mathbf{u})=0
$$

volume fraction

$$
\frac{\partial(\rho \gamma)}{\partial t}+\nabla \cdot(\rho \gamma \mathbf{u})=0
$$

momentum

$$
\frac{\partial}{\partial t}(\rho \mathbf{u})+\nabla(\rho \mathbf{u u})=-\nabla p+\nabla \cdot \mu\left(\nabla \mathbf{u}+\nabla \mathbf{u}^{T}\right)+\rho \mathbf{g}+\mathbf{f}_{\sigma}+\mathbf{f}_{w},
$$

where $\mathbf{u}$ is the fluid velocity, $t$ the physical time, $p$ the pressure, and $\gamma$ the volume fraction of the second phase (gas phase) in the computational cell. When the cell is completely filled with liquid, $\gamma$ equals zero; and $\gamma$ equals one when the cell is completely filled with gas. For a cell containing the interface, $\gamma$ takes a value between 0 and $1 . \mathbf{g}$ is the acceleration due to gravity, $\rho$ the average density, and $\mu$ the average dynamic viscosity. In our case, $\rho$ and $\mu$ are constant in each fluid with a jump at the interface. They can be defined by

$$
\begin{aligned}
& \rho=\gamma \rho_{g}+(1-\gamma) \rho_{l}, \\
& \mu=\gamma \mu_{g}+(1-\gamma) \mu_{l} .
\end{aligned}
$$

$\mathbf{f}_{\sigma}$ denotes an additional surface tension source term. The surface tension along the interface between gas bubble and water is described by the continuum surface force (CSF) model. ${ }^{30}$ The force at the surface can be expressed as a volume force using the divergence theorem, which is added to the momentum equation as a source term

$$
\mathbf{f}_{\sigma}=-\sigma \rho \kappa \nabla \gamma /\left(0.5\left(\rho_{g}+\rho_{l}\right)\right),
$$


where $\sigma$ is the surface tension and $\kappa$ the curvature of the interface, which is defined in terms of the divergence of the unit normal

$$
\kappa=\nabla \cdot(\nabla \gamma /|\nabla \gamma|),
$$

where $\nabla \gamma$ is the gradient of the volume fraction $\gamma$ and $|\nabla \gamma|$ the length of $\nabla \gamma$. The detailed calculation of the gradient of the volume fraction function and curvature can be found in Refs. 31 and 32.

$\mathbf{f}_{w}$ is an additional in-gap wall friction source term, which can be described by Poiseuille's law

$$
\mathbf{f}_{w}=-12 \mu \mathbf{u} / h^{2},
$$

where $h$ is the gap thickness of the cell. It means the additional gap-averaged pressure drop in the Hele-Shaw cell has a linear relationship with the gap-averaged velocity. In simulation, Darcy's law is used to calculate the in-gap wall friction due to the similarity between the Hele-Shaw cell wall resistance and porous media resistance. The equation of Darcy's law for porous media resistance is as follows:

$$
\mathbf{f}=-\mu \mathbf{u} / \alpha
$$

where $\alpha$ is the viscous resistance. By comparing Eq. (8) with Eq. (9), the Hele-Shaw flow can be seen as a two-dimensional flow in porous media, whereby the viscous resistance $\alpha$ in the Hele-Shaw cell is equivalent to

$$
\alpha=h^{2} / 12 .
$$

By using Eq. (10), the bubble behaviour in cells with different gap thicknesses can be simulated by adjusting the viscous resistance values.

\section{B. Simulation strategies}

The commercial computational fluid dynamics (CFD) software ANSYS Fluent 14.0 was used to simulate the motion of a single nitrogen gas bubble rising in a vertical Hele-Shaw cell filled with water. The computational domain consisted of a $50 \mathrm{~mm}$ in width by $300 \mathrm{~mm}$ in height rectangle, whose height was $200 \mathrm{~mm}$ less than the experimental conditions. No difference was found by decreasing the height due to small hydrostatic pressure difference (less than $2 \%$ ). The domain was filled with stationary water and a spherical bubble was initialized in the centre bottom of the domain as shown in Figure 2. For the bubbles with large deformation $(d=8-11 \mathrm{~mm}$ for $h=1.0 \mathrm{~mm})$, left-right asymmetric initial bubble shapes such as semicircle were initialized to avoid bubble break-up. The operating pressure was $1 \mathrm{~atm}$. No-slip boundary conditions were applied at all walls. The initial bubble rise velocity was zero. The bubble was able to develop into a typical shape to reach a terminal velocity within about $0.5 \mathrm{~s}$ and to rise to the liquid surface in 2.0-4.0 s depending on the terminal velocity. The steady-state data $(1.0-2.0 \mathrm{~s})$ were used for comparison. The volume fraction cut-off was $10^{-6}$. The simulated bubble size corresponds to the area enclosed by the contour line where the volume fraction $\gamma=0.5$. The following material properties were included in the Fluent database: the density of the water is $997 \mathrm{~kg} / \mathrm{m}^{3}$, the viscosity of the water is $8.94 \times 10^{-4} \mathrm{~Pa} \mathrm{~s}$; the density of the nitrogen is $1.185 \mathrm{~kg} / \mathrm{m}^{3}$, the viscosity of the nitrogen is $1.663 \times 10^{-5} \mathrm{~kg} /(\mathrm{ms})$. The surface tension is $0.0729 \mathrm{~N} / \mathrm{m}$. Uniform quadrilateral meshing was used, each cell was $0.25 \mathrm{~mm}$ by $0.25 \mathrm{~mm}$, which means 240000 cells in total. Grid independence was proved by further reducing the cell size to $0.125 \mathrm{~mm}$ yielding only a $0.5 \%$ terminal velocity difference. The terminal velocity difference obtained from a laminar model and a turbulent model (large eddy simulation) is small (3\%). A laminar model was chosen due to small cell Reynolds number values.

During the calculation, the unsteady scalar (pressure, velocity components, and volume fraction) transport equations were discretized to algebraic equations by a control-volume method. An explicit geometric reconstruction scheme that is based on the PLIC (Piecewise-Linear-Interface-Calculation) method $^{33}$ was used to solve the volume fraction equation. Second-order upwind scheme was used to discretize the convection terms of the velocity components. The diffusion terms were discretized using a second-order accurate central differencing scheme. The gradients of the scalars needed in the convection and diffusion discretization were calculated using the least squares cell-based method. The 


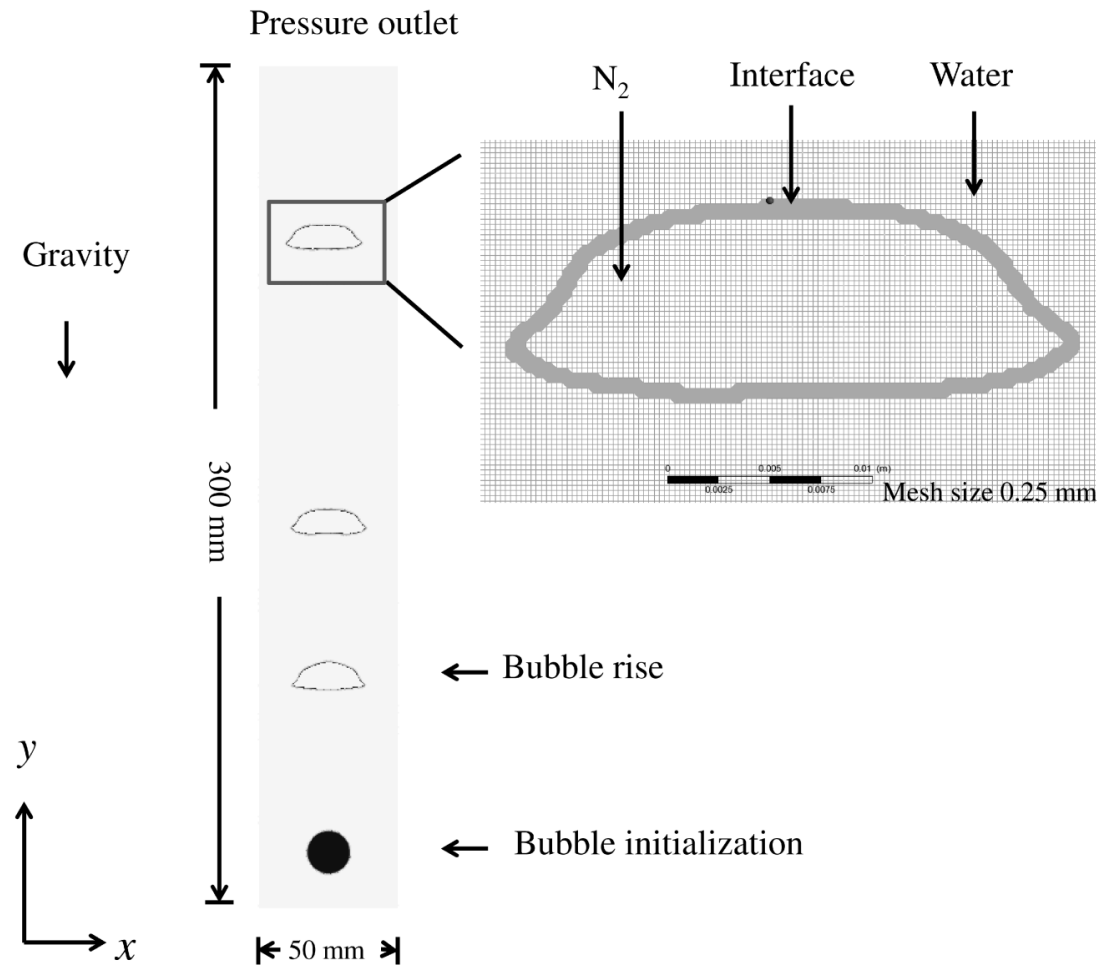

FIG. 2. Computational domain and boundary conditions in simulation.

velocity-pressure coupling and overall solution procedure were based on the SIMPLE (Semi-ImplicitMethod for Pressure Linked Equations) segregated algorithm. A first order implicit time-marching scheme was used for the integration of the transient term. A constant time step $(0.0005 \mathrm{~s})$ was used.

\section{RESULTS AND DISCUSSION}

The bubble behaviour in the Hele-Shaw cell with the gap thicknesses of (1) $h=0.5 \mathrm{~mm}$, viscous resistance $1 / \alpha=4.8 \times 10^{7} \mathrm{~m}^{-2}$; (2) $h=1.0 \mathrm{~mm}$, viscous resistance $1 / \alpha=1.2 \times 10^{7} \mathrm{~m}^{-2}$ was simulated and compared with experimental results. Sections IV A and IV B show the results of $h=0.5 \mathrm{~mm}$ and $h=1 \mathrm{~mm}$, respectively.

\section{A. Results for gap thickness $h=0.5 \mathrm{~mm}$}

In this section, the experimentally observed and simulated results of bubble shape, bubble path, and bubble terminal velocity are compared and evaluated. The vortex and flow distribution are illustrated to understand the bubble dynamics fully.

\section{Bubble shape and path}

Two shape regimes are found in the case of gap thickness $h=0.5 \mathrm{~mm}$ : oblate ellipsoid ( $d$ $<10.5 \mathrm{~mm})$ and spherical-cap $(d>12 \mathrm{~mm})$. The transition from oblate ellipsoid to spherical-cap occurs when $10.5 \mathrm{~mm}<d<12 \mathrm{~mm}$. The upper part of Figure 3 shows some typical experimentally observed bubble shapes. When $d$ is small (Figures 3(a) and 3(b)), the bubble takes the shape of oblate ellipsoid. With an increase of $d$ (Figure 3(c)), the bubble becomes unstable and loses the fore-and-aft symmetry. The fore-part of the bubble tends to have a negative curvature, which results in a transition to a spherical-cap regime (Figure 3(d)). A further increase in $d$ does not change the bubble shape 


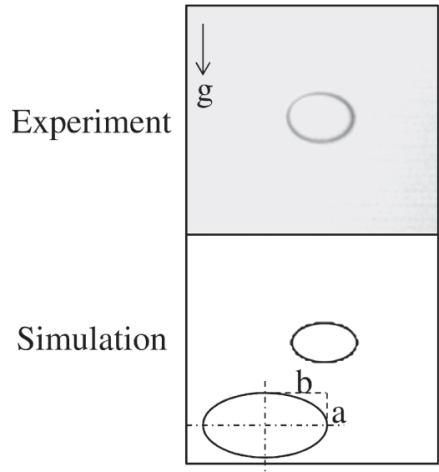

(a)

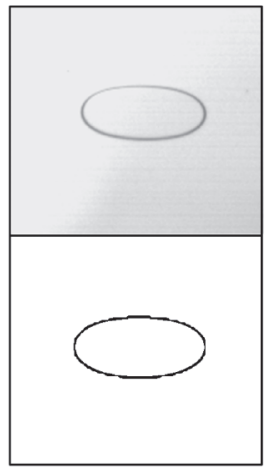

(b)

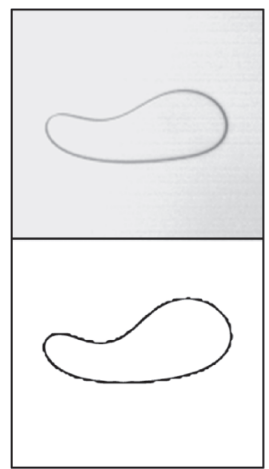

(c)

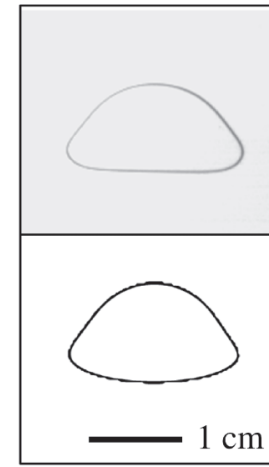

(d)

FIG. 3. Comparison of bubble shapes between experiment and simulation for gap thickness $h=0.5 \mathrm{~mm}$. Top: experiment. Bottom: simulation. (a) $d=5 \mathrm{~mm}$; (b) $d=8 \mathrm{~mm}$; (c) $d=11.5 \mathrm{~mm}$; (d) $d=13 \mathrm{~mm}$. The downward arrow and symbol g show the direction of gravitational force.

anymore. The simulated bubble shapes in the lower part of Figure 3 agree with the observed ones. To further characterize the shape change, the aspect ratio $(E)$ evolution is illustrated in Figure 4, which is the ratio of the length projected on the symmetry axis to the maximum diameter normal to the axis ${ }^{34}$ $(a / b$ in Figure 3). In the oblate ellipsoidal regime, $E$ decreases with the increase of $d$, reaching lowest value of 0.31 . In the spherical-cap regime, $E$ increases with increasing $d$. The simulation results show a similar tendency with small deviations.

The paths of the bubbles are shown in Figure 5. Experiment and simulation show very similar results for different types of bubbles. A rectilinear path is observed for the oblate ellipsoidal and spherical-cap bubbles. The path deviates only when the shape undergoes the transition from oblate ellipsoid to spherical-cap (Figure 5(c)). The path deviation lasts for less than $0.1 \mathrm{~s}$. Once the transition is finished, the path becomes rectilinear again.

\section{Bubble terminal velocity}

Figure 6 compares the simulated and observed terminal velocity for steady-state shapes. The observed velocity first increases and then decreases in the oblate ellipsoidal regime. In this regime, when the bubble size is small $(d<5 \mathrm{~mm})$, the increased buoyancy force leads to a velocity increase.

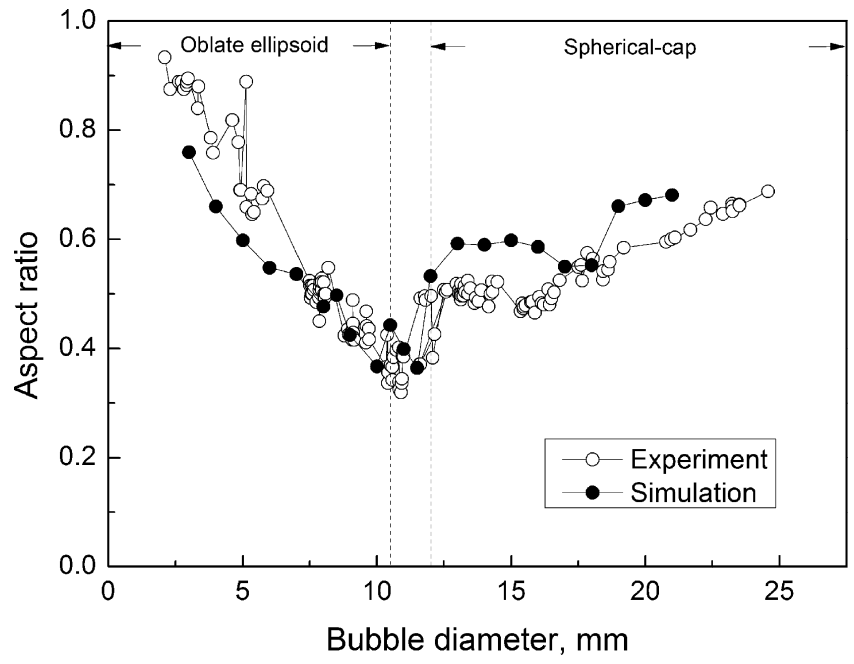

FIG. 4. Comparison of bubble aspect ratio between experiment and simulation for gap thickness $h=0.5 \mathrm{~mm}$. 


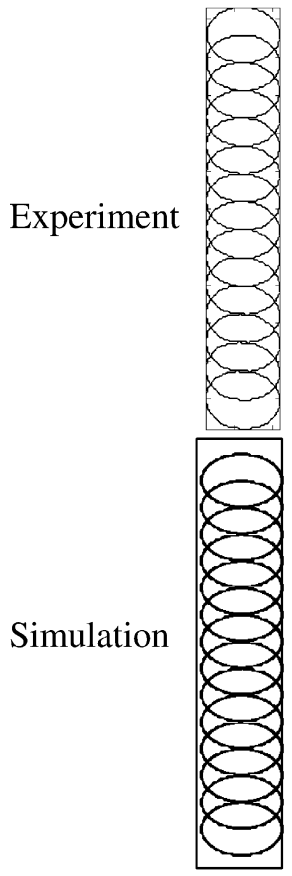

(a)

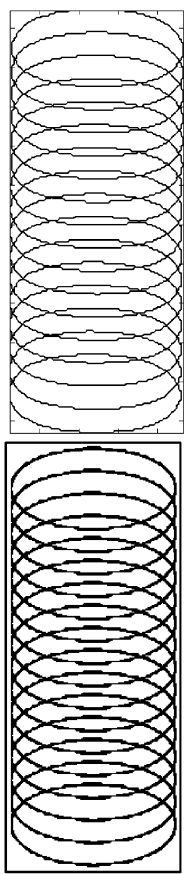

(b)

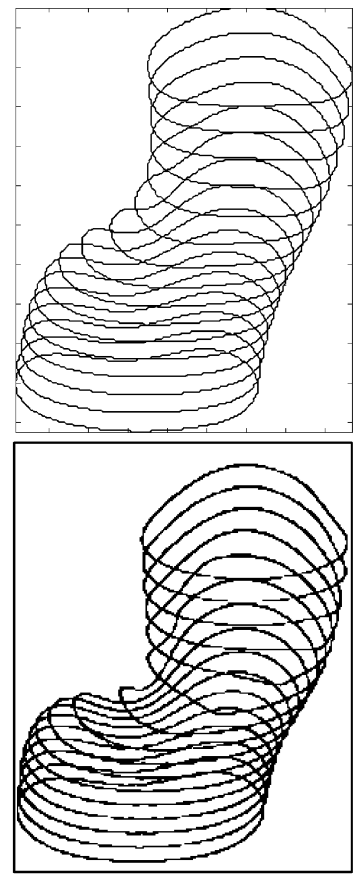

(c)

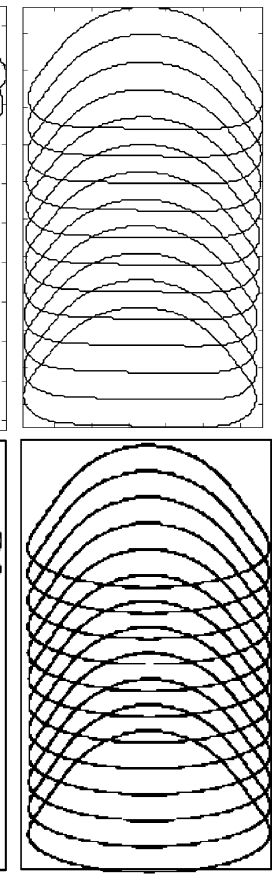

(d)

FIG. 5. Comparison of bubble paths between experiment and simulation for gap thickness $h=0.5 \mathrm{~mm}$. The successive contours are plotted at a time interval of $0.02 \mathrm{~s}$. Top: experiment. Bottom: simulation. (a) $d=5 \mathrm{~mm}$; (b) $d=8 \mathrm{~mm}$; (c) $d=11.5 \mathrm{~mm}$; (d) $d=13 \mathrm{~mm}$.

With a further increase in bubble size, the drag force is increased due to the increase of the aspect ratio and therefore results in a decrease in terminal velocity. For transition bubbles, the terminal velocity increases with the bubble size as the aspect ratio increases. The change of terminal velocity becomes limited when the bubble reaches a spherical-cap shape $(d>12 \mathrm{~mm})$. The overall experimental terminal velocity range is $0.046-0.10 \mathrm{~m} / \mathrm{s}(d=2-25 \mathrm{~mm})$. Compared to the experimental data, the simulation results follow a similar trend as a function of bubble size when $d>5 \mathrm{~mm}$. However, the simulation overestimates the terminal velocity when $d \leq 5 \mathrm{~mm}$. The smaller the bubble size, the higher the deviation $(10 \%-50 \%)$ between experiment and simulation.

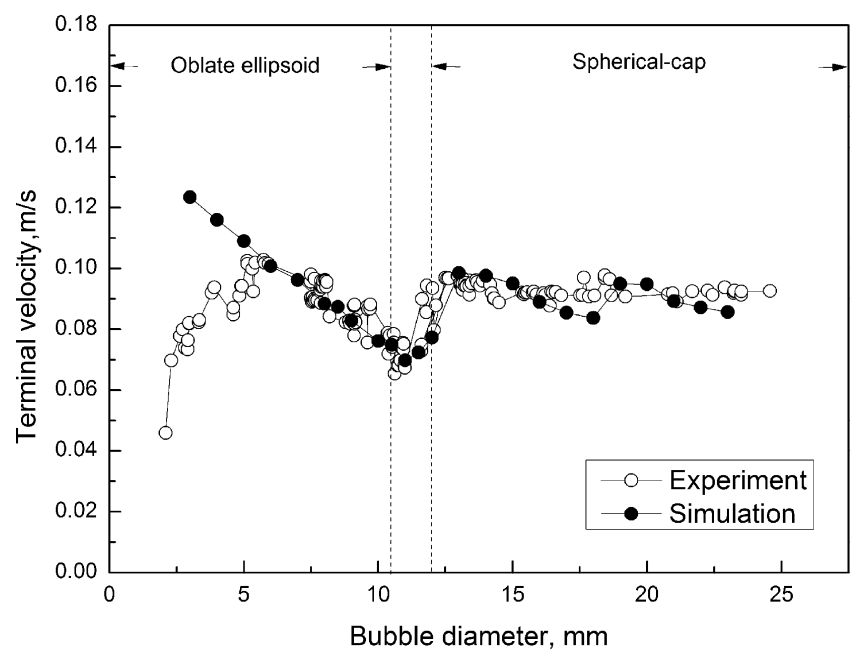

FIG. 6. Comparison of terminal velocity between experiment and simulation for gap thickness $h=0.5 \mathrm{~mm}$. 


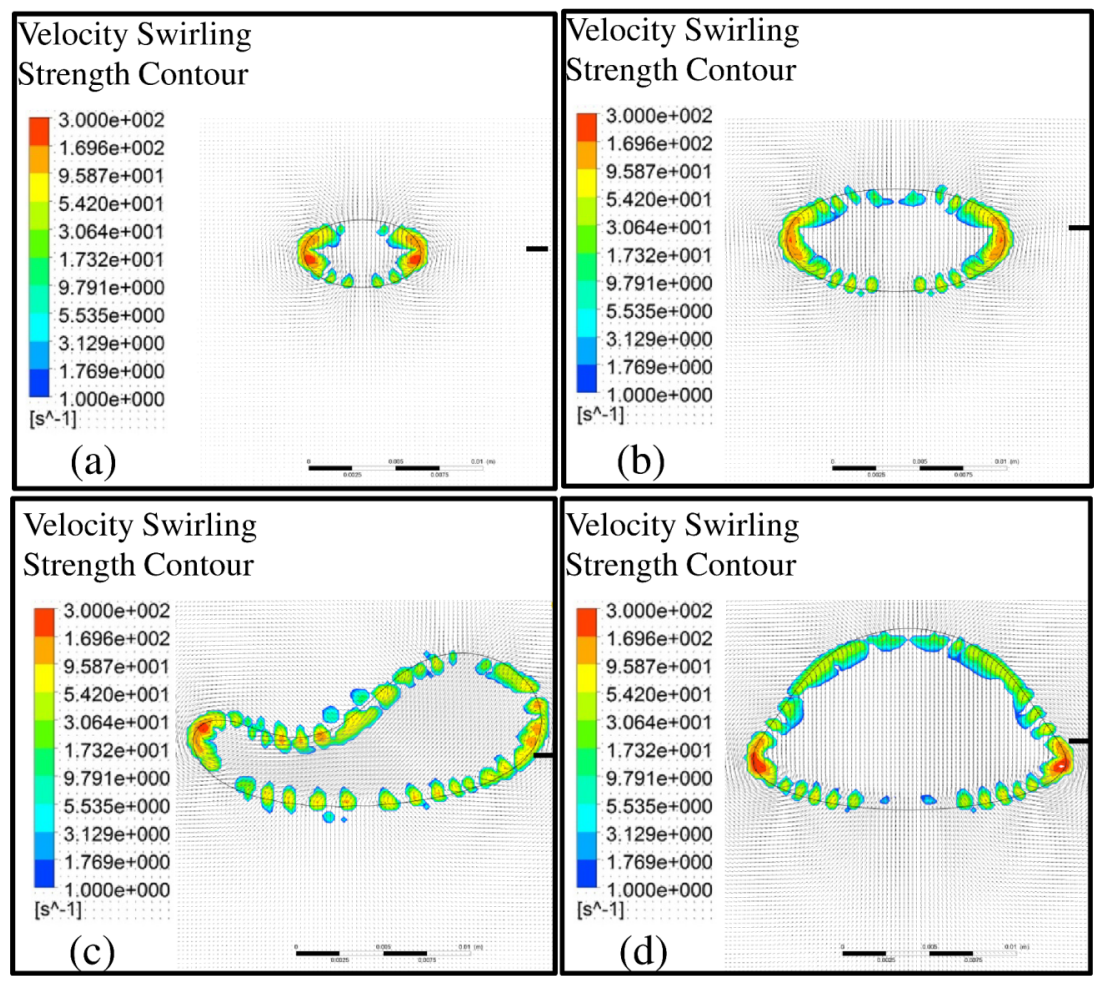

FIG. 7. The simulated velocity swirling strength contours for gap thickness $h=0.5 \mathrm{~mm}$. (a) $d=5 \mathrm{~mm}$; (b) $d=8 \mathrm{~mm}$; (c) $d=11.5 \mathrm{~mm}$; (d) $d=13 \mathrm{~mm}$.

\section{Vortex and flow distribution}

The simulated vortices are given in Figure 7 to further understand the bubble path. The swirling strength $^{35}$ is used to identify and visualize the vortex contour, which uses the imaginary part of the complex eigenvalue of $\nabla \mathbf{u}$ to visualize vortices and to quantify the strength of the local swirling motion inside the vortex. ${ }^{36}$ It can be seen that the vortices are found only near the gas bubble/water interface. This is due to the spurious vortical currents induced by the coupled CSF force model.$^{31}$ It is a common problem in simulation and does not affect the calculation of bubble shape and terminal velocity. 37,38 Vortices are not found in the liquid, which means that the vorticity is not strong enough to form a vortex core, and the wake is thus closed without vortex shedding. Without the effect of vortices, the path orientation is not perturbed and remains rectilinear.

The vertical velocity across the bubble interface along the whole cell width is illustrated in Figure 8. In all cases, the liquid backflow can be seen clearly. Except for $d=5 \mathrm{~mm}(d / W \leq 0.1)$, the no-slip boundary conditions will directly affect the flow profile in the plane of the cell.

To summarize, the following flow behaviour can be obtained from experiments for gap thickness $h=0.5 \mathrm{~mm}$ : (1) two shape regimes when $d \geq 2 \mathrm{~mm}$, oblate ellipsoid and spherical-cap; (2) oblate ellipsoidal and spherical-cap bubbles take the rectilinear path; shape transition results in path deviation, the deviation lasts for $0.1 \mathrm{~s}$; (3) the terminal velocity is in the range of $0.46-0.10 \mathrm{~m} / \mathrm{s}$. The simulation results agree with the experimental results very well except for the overestimation of the terminal velocity when $d \leq 5 \mathrm{~mm}$. The simulation shows no vortices in the liquid region, which explains the rectilinear path. It also shows that the side-wall effects are not negligible when $d / W<0.1$.

\section{B. Results for gap thickness $h=1.0 \mathrm{~mm}$}

Similar to Sec. IV A, the experimental and simulated results on bubble shape, bubble path, bubble terminal velocity, and bubble secondary motion are compared in this section for a gap thickness of $h=1.0 \mathrm{~mm}$, followed by the simulated results on vortex and flow distribution. 


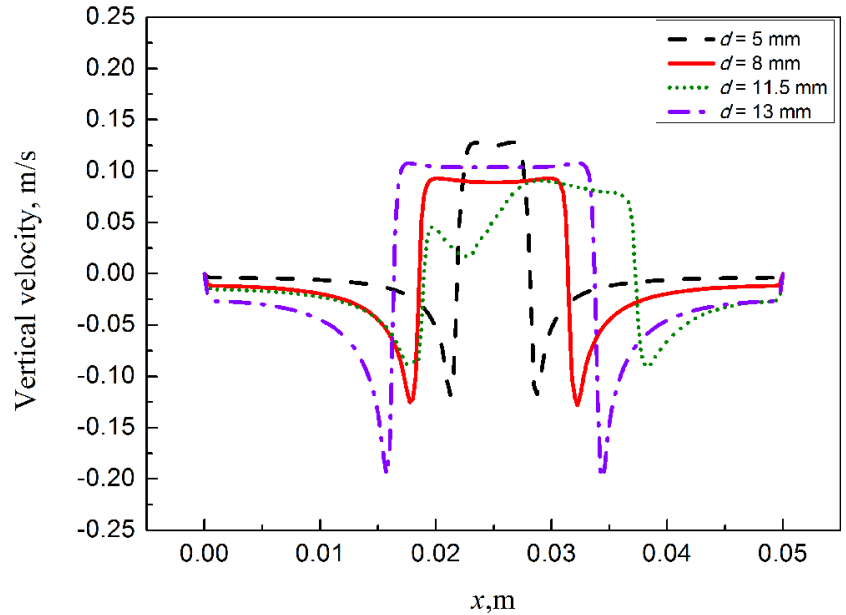

FIG. 8. The vertical velocity distribution as a function of horizontal distance $x$ for gap thickness $h=0.5 \mathrm{~mm}$. The height at which the $x$ coordinate axis is positioned is marked with a short marker line on the right side of the plots in Figure 7.

\section{Bubble shape and path}

The bubble shapes for gap thickness $h=1.0 \mathrm{~mm}$ are more complicated compared with those for $h=0.5 \mathrm{~mm}$. Except for the shapes of oblate ellipsoid and spherical-cap, bubble rotation, deformation, and oscillation (periodic deformation) result in two more shape regimes: a wobbling regime and an oscillating spherical-cap regime. The typical contours of the four shape regimes are illustrated in Figure 9. Figure 9(a) illustrates an oblate ellipsoid bubble. Its fore-aft and left-right symmetry is lost due to shape rotation. Figure 9(b) is a wobbling bubble. The interface of the bubble is deformed by shape oscillation. The oscillating spherical-cap bubble contour is shown in Figure 9(c), which has left-right symmetry as the spherical-cap bubble (Figure 9(d)). The simulated bubble shapes (bottom part of Figure 9) are very close to the observed bubble shapes and agree with the results in Refs. 13 and 16.

The comparison of the typical bubble paths is shown in Figure 10. Different bubble regimes have different bubble paths. The oblate ellipsoidal bubble (Figure 10(a)) has a zigzag path with a very small shape oscillation. The wobbling bubble (Figure 10(b)) has a zigzag path with a strong shape oscillation. For oscillating spherical-cap bubble (Figure 10(c)), the path becomes rectilinear accompanied by a continuous shape oscillation. The shape oscillation disappears for a spherical-cap bubble (Figure 10(d)), resulting in a straight path only. The simulation can predict the path transition and shape change correctly. Differently, the simulated spherical-cap bubble $(d=20 \mathrm{~mm})$ has a small fluctuation in the rear surface, which disappears when $d \geq 22 \mathrm{~mm}$.

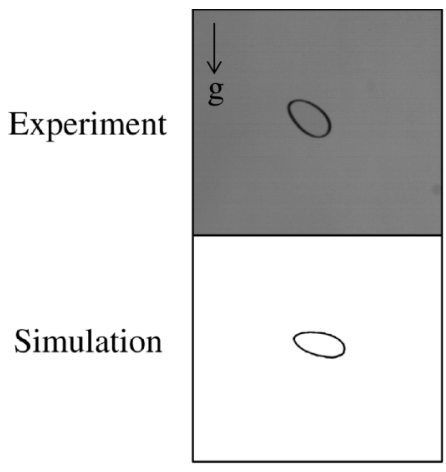

(a)

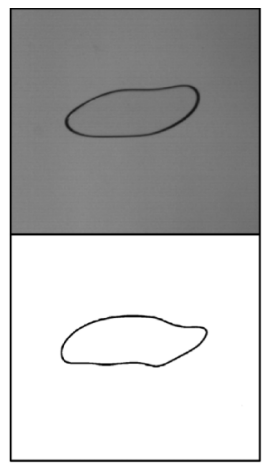

(b)

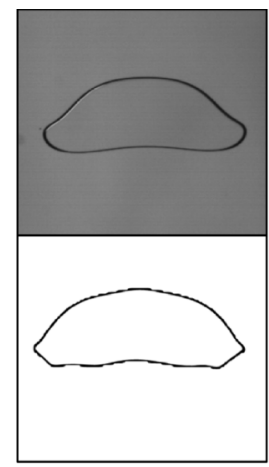

(c)

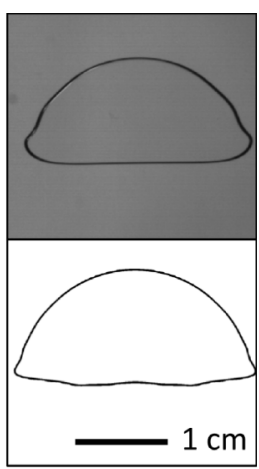

(d)

FIG. 9. Comparison of bubble shapes between experiment and simulation for gap thickness $h=1.0 \mathrm{~mm}$. Top: experiment. Bottom: simulation. (a) $d=5 \mathrm{~mm}$; (b) $d=10 \mathrm{~mm}$; (c) $d=15 \mathrm{~mm}$; (d) $d=20 \mathrm{~mm}$. The downward arrow and symbol g stand for the direction of gravitational force. 

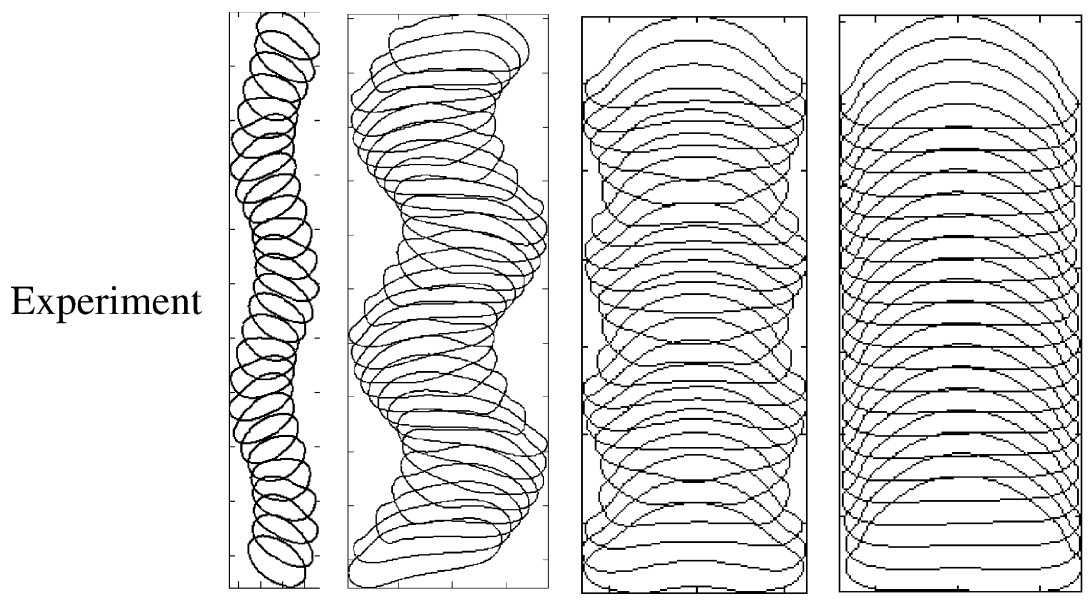

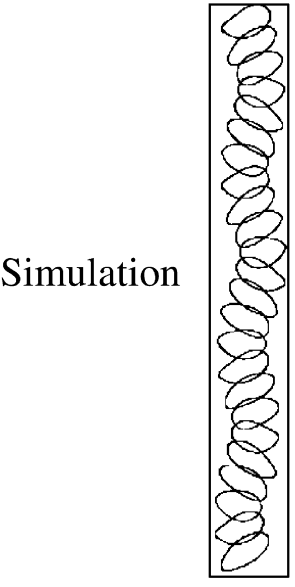

(a)

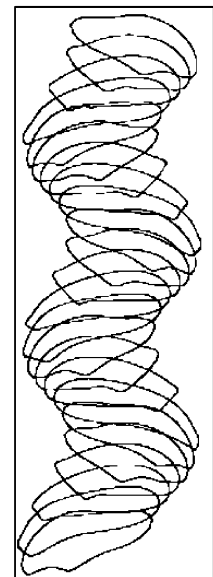

(b)

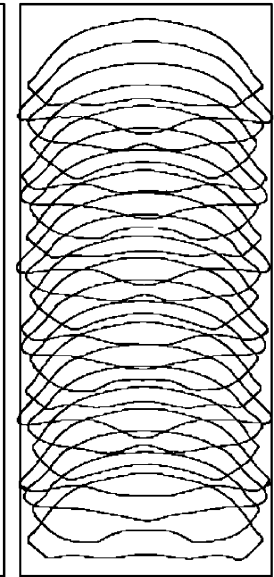

(c)

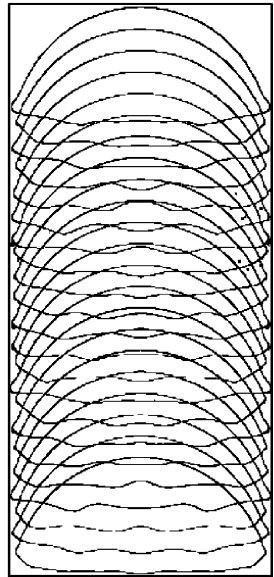

(d)

FIG. 10. Comparison of bubble paths between experiment and simulation for gap thickness $h=1.0 \mathrm{~mm}$. The successive contours are plotted at a time interval of $0.02 \mathrm{~s}$. Top: experiment. Bottom: simulation. (a) $d=5 \mathrm{~mm}$; (b) $d=10 \mathrm{~mm}$; (c) $d=15 \mathrm{~mm}$; (d) $d=20 \mathrm{~mm}$.

\section{Bubble terminal velocity}

The comparison of the terminal velocity in experiment and simulation is illustrated in Figure 11. Since there are four shape regimes now, the terminal velocity evolution as a function of bubble size is more complicated than that of $h=0.5 \mathrm{~mm}$ (two regimes). In the experiment, the terminal velocity increases with increasing bubble size when the bubble is oblate ellipsoidal due to the increased buoyancy force. The terminal velocity first decreases and then increases when the bubble is wobbling. It reaches the lowest value when $d=10.5 \mathrm{~mm}$. The velocity decreases again when the bubble is in the oscillating spherical-cap regime due to energy dissipation during oscillation. The terminal velocity increases when the spherical-cap regime is reached due to the decrease of the shape oscillation, and it becomes almost constant in the end. The overall terminal velocity range is $0.10-0.14 \mathrm{~m} / \mathrm{s}$. The simulated bubble terminal velocity is close to the experimental results with an averaged $9 \%$ deviation (higher value in simulation) when $d \leq 18 \mathrm{~mm}$.

\section{Horizontal vibration}

The zigzag path is caused by the periodic horizontal vibration. To describe the horizontal vibration of the bubbles, a sine function similar to the one proposed by Kozuka et al. ${ }^{14}$ can be used

$$
V_{x}(t)=V_{0} \sin (\omega t+\alpha),
$$




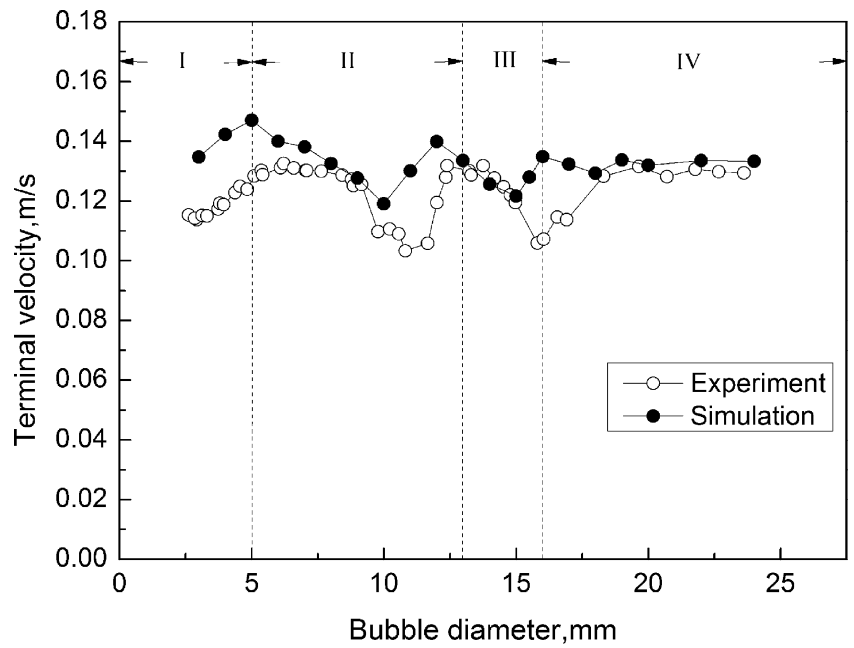

FIG. 11. Comparison of terminal velocity for gap thickness $h=1.0 \mathrm{~mm}$. I: oblate ellipsoid; II: wobbling; III: oscillating spherical-cap; IV: spherical-cap.

where $V_{x}(t)$ is the horizontal velocity at a given time $t, V_{0}$ the horizontal velocity amplitude, $\omega=2 \pi f_{1}$ the angular frequency, and $\alpha$ the initial phase difference. Therefore, two important parameters, namely amplitude $V_{0}$ and frequency $f_{1}$, can be used to characterize the horizontal vibration. Their experimental and theoretical comparisons are shown in Figure 12. It can be seen from Figure 12(a) that only oblate ellipsoidal and wobbling bubbles have periodic horizontal velocity. The observed horizontal velocity amplitude decreases with increasing bubble size when the bubble is oblate ellipsoidal, then it increases when the bubble starts wobbling and finally decreases to zero when the bubble becomes spherical-cap. The highest value of $0.09 \mathrm{~m} / \mathrm{s}$ is reached when $d=10.5 \mathrm{~mm}$. The horizontal vibration evolution goes the contrary to the evolution of the terminal velocity. The simulated amplitude evolution follows a similar tendency as in the experiments but with a higher peak value. Figure 12(b) shows the frequency comparison. It is clear that the experimentally horizontal velocity frequency is in the range of 4-6 Hz. The simulated frequency has a similar change but with a higher frequency range of $5-8 \mathrm{~Hz}$.

\section{Shape oscillation}

Similar to the horizontal vibration, the shape oscillation can also be described by amplitude and frequency. Perimeter oscillation is used in this study to describe the bubble shape oscillation. Only the frequency with the larger amplitude is compared. Figure 13(a) shows the perimeter oscillation

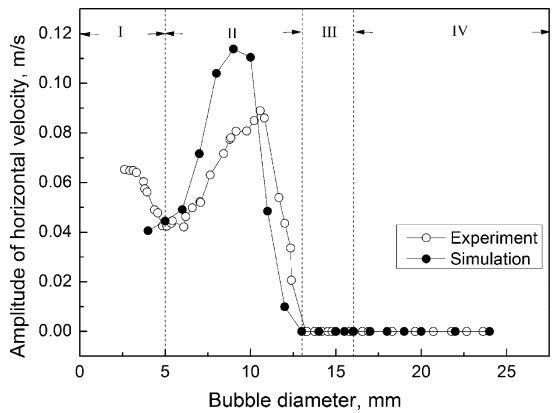

(a)

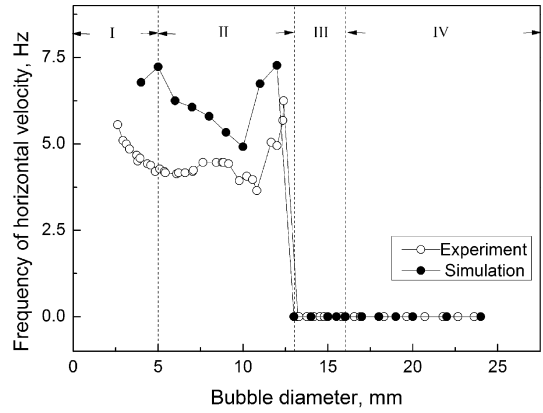

(b)

FIG. 12. Comparison of horizontal velocity (a) amplitude and (b) frequency for gap thickness $h=1.0 \mathrm{~mm}$. I: oblate ellipsoid; II: wobbling; III: oscillating spherical-cap; IV: spherical-cap. 


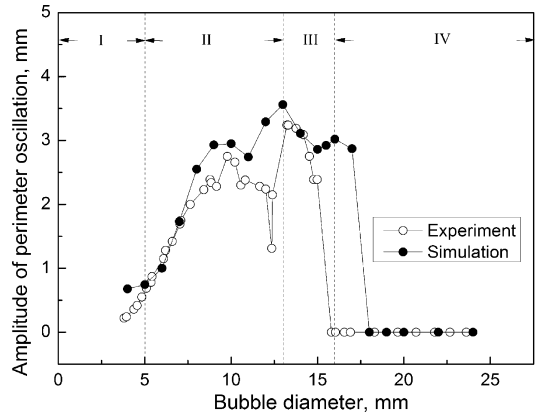

(a)

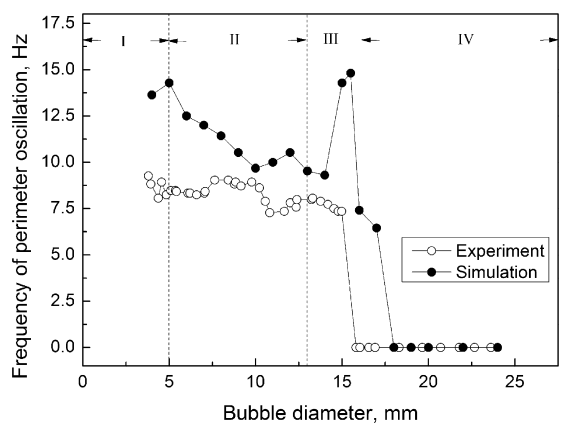

(b)

FIG. 13. Comparison of perimeter oscillation (a) amplitude and (b) frequency for gap thickness $h=1.0 \mathrm{~mm}$. I: oblate ellipsoid; II: wobbling; III: oscillating spherical-cap; IV: spherical-cap.

amplitude as a function of bubble size. The oscillation shows up in three bubble types. The effect is very small when the bubble is oblate ellipsoidal. When the bubble is wobbling, the amplitude first increases and then decreases to a lowest value. The oscillation slows down gradually until it reaches zero when the oscillated bubble is spherical-cap. The simulated results follow the same evolution as a function of bubble size. However, when $16 \leq d \leq 17 \mathrm{~mm}$, the simulated bubbles still have shape oscillation with a constant frequency while the experimentally observed bubbles do not.

Figure 13(b) shows the frequency comparison of the perimeter oscillation. The frequency of the perimeter oscillation in the experiments is approximately twice the frequency of the horizontal vibration when $d \leq 10.5 \mathrm{~mm}$, then the shape oscillation frequency becomes independent due to the reduced path vibration. The predicted frequency in the simulations is also higher than the observed one. Irregularity is found in the simulation results when $d=15$ and $15.5 \mathrm{~mm}$, with a frequency that is

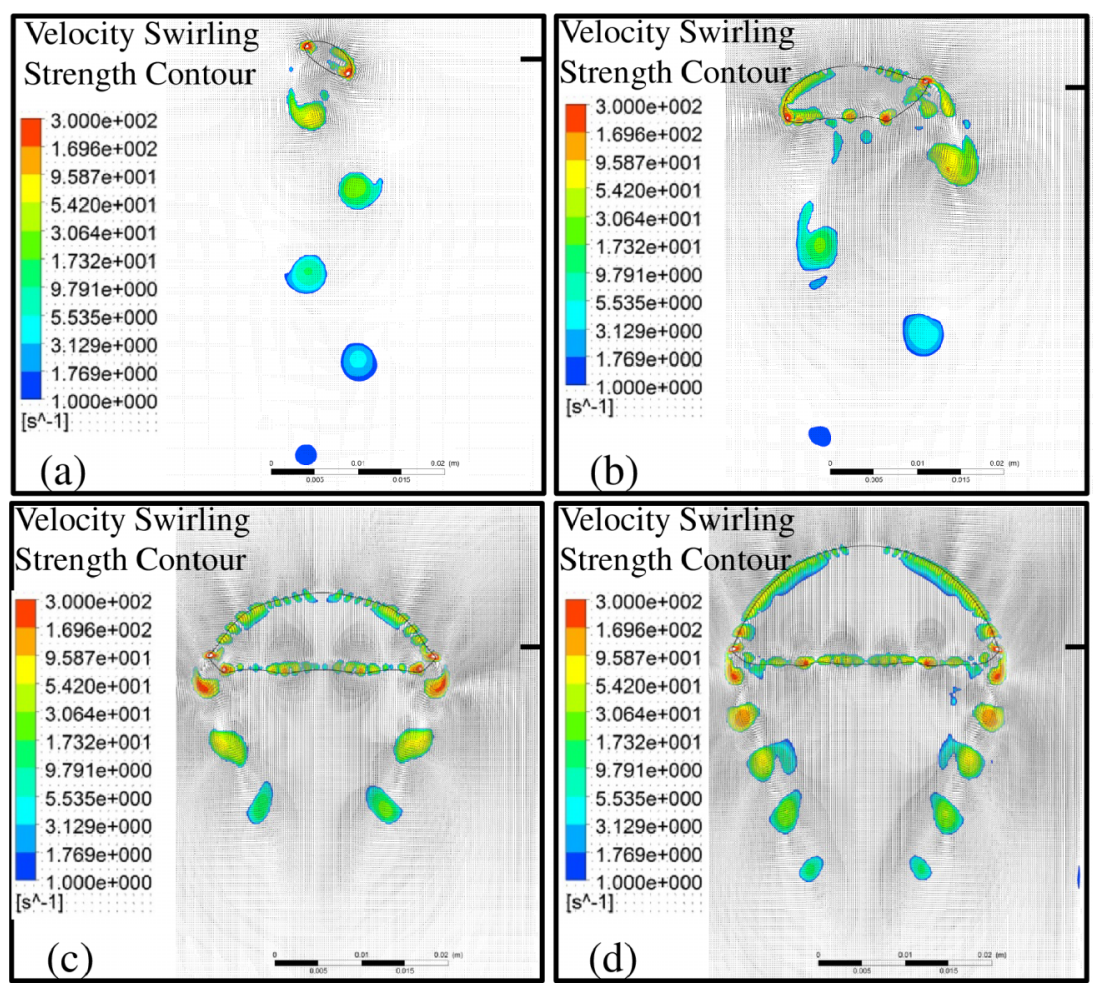

FIG. 14. The simulated velocity swirling strength contours for gap thickness $h=1.0 \mathrm{~mm}$. (a) $d=5 \mathrm{~mm}$; (b) $d=10 \mathrm{~mm}$; (c) $d=15 \mathrm{~mm}$; (d) $d=20 \mathrm{~mm}$. 


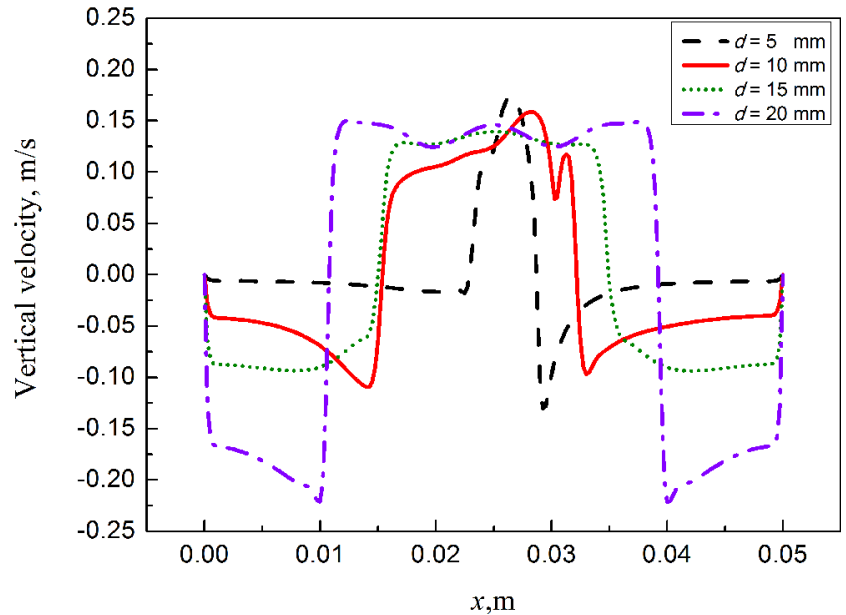

FIG. 15. The vertical velocity distribution as a function of horizontal distance $x$ for gap thickness $h=1.0 \mathrm{~mm}$. The height at which the $x$ coordinate axis is positioned is marked with a short marker line on the right side of the plots in Figure 14.

twice that of the neighbouring bubbles. The reason for this is not clear, it is possible that the secondary oscillation is suddenly enlarged so that a higher magnitude as the primary oscillation is obtained.

\section{Vortex and flow distribution}

The simulated flow vortex is illustrated in Figure 14 to give a better understanding of the gasliquid flow interaction. The same vortex detection method is used as in the case of gap thickness $h$ $=0.5 \mathrm{~mm}$. Different from that case, vortex shedding is found now for all bubble types and it is affected by the bubble regimes. For oblate and wobbling bubbles which go through a zigzag path, the vortices are asymmetric. For spherical-cap bubbles with a rectilinear path, vortices are shedding simultaneously from both sides of the bubble edge. The dissipation of the vortex is very fast. Only a few vortices are found behind the bubble. The simulation results resemble the experimental observation in Ref. 14.

Similarly, the simulated vertical velocity distribution across the bubble interface along the whole width is shown in Figure 15. The liquid velocity near the side walls is very high, and increases with an increase of the bubble size. When $d=20 \mathrm{~mm}$, the liquid backflow velocity is about $0.15 \mathrm{~m} / \mathrm{s}$ near the wall. This means that the side-wall effects are influential to the bubble terminal velocity and shape oscillation.

As a whole, the bubble behaviour in a cell with gap thickness $h=1.0 \mathrm{~mm}$ has the following characteristics: (1) four bubble shapes, oblate ellipsoid, wobbling, oscillating spherical-cap, and stable spherical-cap; (2) in addition to a rectilinear path, a zigzag path is coupled with the oscillating bubble shape; (3) the bubble terminal velocity is in the range of $0.10-0.14 \mathrm{~m} / \mathrm{s}$; (4) the horizontal vibration affects two bubble regimes, while the shape oscillation affects three bubble regimes. All these phenomena can be simulated reasonably well.

\section{Discussion}

\section{Comparison of the bubble behaviour in the two gap thicknesses}

As shown in the results, by changing the cell gap thickness, the bubble flow characteristics including shape, path, terminal velocity, and vortex become quite different, as summarized in Table I. Only two stable shapes are found in the case of gap thickness $h=0.5 \mathrm{~mm}$, while four are present in the case of $h=1.0 \mathrm{~mm}$. Furthermore, shape oscillation accompanies the wobbling and spherical-cap regimes of $h=1.0 \mathrm{~mm}$. In the current experiments, the bubble size and cell width are kept the same with identical $A r$ and $B o$, and therefore any difference in the bubble motion behaviour is directly caused by 
TABLE I. The vertical Hele-Shaw bubble motion in two different gap thicknesses in the experiment (vortex from simulation).

\begin{tabular}{lcllll}
\hline \hline$h, \mathrm{~mm}$ & $d, \mathrm{~mm}$ & \multicolumn{1}{c}{ Shape } & \multicolumn{1}{c}{ Path } & Terminal velocity, m/s & \multicolumn{1}{c}{ Vortex } \\
\hline 0.5 & $2-10.5$ & Oblate ellipsoid & Rectilinear & $0.46-0.10$ & No \\
0.5 & $12-25$ & Spherical-cap & Rectilinear & 0.10 & No \\
1.0 & $2-5$ & Oblate ellipsoid & Zigzag & $0.11-0.14$ & Asymmetric shedding \\
1.0 & $5-13$ & Wobbling & Zigzag & $0.11-0.14$ & Asymmetric shedding \\
1.0 & $13-16$ & Oscillating spherical-cap & Rectilinear & $0.11-0.13$ & Symmetric shedding \\
1.0 & $16-25$ & Spherical-cap & Rectilinear & 0.13 & Symmetric shedding \\
\hline
\end{tabular}

the change in the gap thickness. With a narrow gap thickness, a higher viscous resistance is expected and the dependence of bubble motion on bubble size can also be less significant. As given in Table I, the bubble paths for $h=0.5 \mathrm{~mm}$ are rectilinear only, while both zigzag and rectilinear paths can be observed for $h=1.0 \mathrm{~mm}$ in the observed bubble size range. A difference in the corresponding terminal velocity is also observed. A higher velocity is obtained for $h=1.0 \mathrm{~mm}$ for the same bubble size. In addition, different gap thicknesses result in very distinctive vortex behaviour: no vortices when $h=0.5 \mathrm{~mm}$, while vortex shedding and the ensuing bubble oscillation when $h=1.0 \mathrm{~mm}$.

\section{Governing parameters for the bubble shape and path instabilities}

The non-dimensional governing parameters for the unbounded two-dimensional bubble dynamics in a vertical Hele-Shaw cell include $A r, B o$, and $h / d$. In this research, although the cell width is finite, $r_{c} / W<0.7$ is satisfied in the whole bubble size range, with a maximum value of 0.6 . Therefore, the effect of $h / d$ is significant and cannot be simply replaced by $h / W$. That is to say, the dimensionless numbers for our finite bubble dynamics include $A r, B o, h / d$, and $d / W$. To quantify how these parameters control the bubble dynamics, especially to discover the onset of the shape and path instabilities, the $R e$ and $\operatorname{Re}(h / d)^{2}$ evolution as a function of $A r$ is compared in Figure 16. For generalization, the experimental results of the unconfined bubbles in Ref. 16 are also presented to compare with our results.

The effects of $d / W$ can be seen by comparing the results of $h=1.0 \mathrm{~mm}, W=50 \mathrm{~mm}$ with those of $h=1.0 \mathrm{~mm}, W=400 \mathrm{~mm}$ by Roig et al. ${ }^{16}$ When $h=1.0 \mathrm{~mm}, W=50 \mathrm{~mm}$, there is a critical $A r$ number $\left(A r_{1}=7500\right)$ which controls the disappearance of the shape and path instabilities. For $W=400 \mathrm{~mm}$, the critical $A r$ number increases to $A r_{2} \approx 10000 .{ }^{16}$ Besides, higher $R e$ and $\operatorname{Re}(h / d)^{2}$ values are observed when $A r \geq 2500$. Despite the difference in behaviour, it is clear that the shape and path instabilities are not controlled by $d / W$ when $A r<7500$ since different $d / W s$ can have similar bubble instabilities. Instead, $h / d$ is more likely to be the main controlling parameter due to the distinct behaviour in the two different gap thicknesses. When $h=0.5 \mathrm{~mm}$, there is no

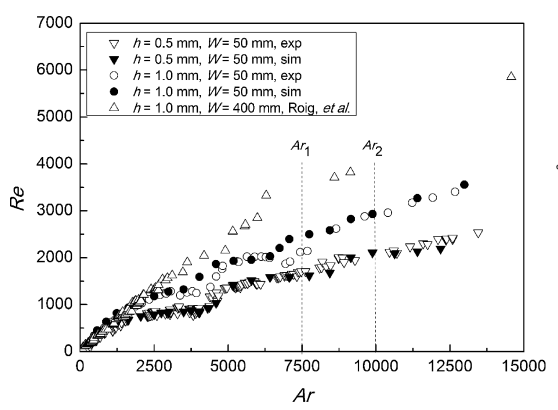

(a)

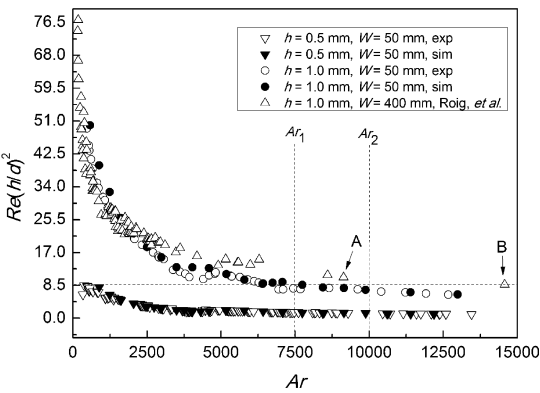

(b)

FIG. 16. $\operatorname{Re}$ and $\operatorname{Re}(h / d)^{2}$ evolution as a function of $A r$. (a) $\operatorname{Re}$; (b) $\operatorname{Re}(h / d)^{2}$. The values for $\operatorname{Re}(h / d)^{2}$ at point $\mathrm{A}$ and $\mathrm{B}$ are 10.6 and 8.1 , respectively. 
critical $A r$ number since all bubbles have a stable shape and path. Therefore, the $A r$ number is not the universal controlling parameter for the shape and path instabilities.

For both gap thicknesses, $R e$ increases linearly with $A r$, following the same trend in Ref. 16. Re falls in the range of 200-2600 and 200-3500 in the case of gap thicknesses $h=0.5$ and $1.0 \mathrm{~mm}$, respectively, when the cell width $W=50 \mathrm{~mm}$. Since high $R e$ motion is obtained in all cases, the $R e$ number by itself cannot explain the observed behavioural differences.

The $\operatorname{Re}(h / d)^{2}$ evolution is shown in Figure 16(b). For $h=0.5 \mathrm{~mm}, W=50 \mathrm{~mm}$, all bubbles have a stable shape and path and they lie in the region where $\operatorname{Re}(h / d)^{2}<8.5$. For $h=1.0 \mathrm{~mm}$, $W=50 \mathrm{~mm}$, the stable bubbles when $A r_{1} \geq 7500$ also lie in the same region of $\operatorname{Re}(h / d)^{2} \leq 8.5$. For a wide cell of $h=1.0 \mathrm{~mm}, W=400 \mathrm{~mm}$, the transition between oscillatory and steady motions is hard to define experimentally due to the difficulty of producing a single bubble. ${ }^{16}$ However, based on the available two data points (A and B in Figure 16(b), their trajectories were shown in Figure 13, Ref. 16), it was found that the bubble is unstable when $\operatorname{Re}(h / d)^{2}=10.6$ (point A) while it is stable when $\operatorname{Re}(h / d)^{2}=8.1$ (point B), therefore, a similar $\operatorname{Re}(h / d)^{2}$ value $\left(8.1 \leq \operatorname{Re}(h / d)^{2}<10.6\right)$ is assumed to control the shape and path instabilities as well. These analogous results demonstrate that $\operatorname{Re}(h / d)^{2}$ determines (or partially determines) the stability of shape and path. Above a critical $\operatorname{Re}(h / d)^{2}$ value, shape oscillation coupled with path vibration determines the main flow characteristics, while shape and path stabilities can be reached once $\operatorname{Re}(h / d)^{2}$ is below this critical value. And the critical value of 8.5 can be applied in both gap thicknesses. Therefore, a general rule based on both the confined bubbles and also the unconfined bubbles can be stated here: small $\operatorname{Re}(h / d)^{2}$ leads to shape and path stabilities.

\section{Model evaluation}

The effectiveness of the gap-averaged 2D simulation is evaluated based on the comparison between simulation and experiment in the two cases. For the case of $h=0.5 \mathrm{~mm}$, except for the overestimation of the terminal velocity when $d \leq 5 \mathrm{~mm}(h / d \geq 0.1)$, the simulated bubble shape, path, and terminal velocity are quite accurate. This good agreement with experiment shows that the $2 \mathrm{D}$ simulation is enough to describe the bubble dynamics when the gap Reynolds number is small. It also confirms that the effects of the thin film effect on the bubble shape and terminal velocity are limited and can be neglected. For the case of $h=1.0 \mathrm{~mm}$, the bubble shape and path are accurate, the terminal velocity can be predicted with an average overestimation of $9 \%$ when $d \leq 18 \mathrm{~mm}(h / d \geq 0.06)$, the secondary motion (path vibration and shape oscillation) can be captured by simulation with a higher frequency. That is to say, the main discrepancy in simulation is the overestimation of the terminal velocity and the oscillation frequency. The overestimation of the terminal velocity in the two cases can be caused by several factors: (1) existence of three-dimensional effects; (2) roughness of the cell, and (3) possible existence of turbulence. Each of these three factors induces a higher viscous dissipation resulting in a low terminal velocity. However, (2) and (3) are not likely to be the main reason because their effects are universal while the overestimation only happens in some size range. Instead, the three-dimensional effects can explain the distinction. When the gap thickness is small $(h=0.5 \mathrm{~mm})$, the three-dimensional effects are minor, so only small bubbles are affected. With a doubling of the gap thickness $(h=1.0 \mathrm{~mm})$, the bubble projected area in the gap is therefore also doubled, so more bubbles are influenced. Especially when the bubble is oscillating, the third-dimensional fluctuation may cause additional energy dissipation. However, the reason for obtaining a higher frequency in the simulation is still not clear, probably the three-dimensional effects contribute to it. In the experiment the constraints of the front and back walls may change the wake structure and slow down the oscillation, which makes the frequency different from the 2D simulation conditions. Apart from the three-dimensional effects, the spurious vortex in the CSF method itself may be a cause of the discrepancy as well. To fully understand the bubble behaviour in the third dimension and its effects, a full $3 \mathrm{D}$ simulation is necessary. 


\section{CONCLUSION}

The bubble dynamics in a vertical Hele-Shaw cell with two different gap thicknesses has been examined experimentally. The main bubble properties including shape, path, and terminal velocity change considerably when the cell gap thickness was narrowed from $h=1.0 \mathrm{~mm}$ to $h=0.5 \mathrm{~mm}$. The shape and path stabilities were found to be determined by a critical value of $\operatorname{Re}(h / d)^{2}$, that is equal to 8.5 under the present experimental conditions. The shape and path are stable when $\operatorname{Re}(h / d)^{2} \leq 8.5$, while vibrating when $\operatorname{Re}(h / d)^{2}>8.5$.

Meanwhile, a 2D VOF method coupled with a CSF model and a wall friction model has been applied to simulate the bubble dynamics in the two cases. By comparing with the experimental results, the simulation results are quantified as follows: (1) For $h=0.5 \mathrm{~mm}$, the bubble shape, bubble path, and bubble terminal velocity can be simulated very well when $d \geq 5 \mathrm{~mm}$. (2) For $h=1.0 \mathrm{~mm}$, the bubble shape and bubble path can also be simulated accurately. The calculated terminal velocity is around $9 \%$ higher when $d \leq 18 \mathrm{~mm}$. The simulated velocity frequency and the perimeter oscillation frequency are $50 \%$ higher than the experimentally observed value. The agreement of the terminal velocity for most of the bubbles in $h=0.5 \mathrm{~mm}$ and large bubbles in $h=1.0 \mathrm{~mm}$ confirms that the thin liquid films have a limited effect on the bubble dynamics and can be neglected. The overestimation of terminal velocity and oscillation frequency is probably due to three-dimensional effects and spurious vortex.

\section{ACKNOWLEDGMENTS}

This work was supported by the Fund for Scientific Research-Flanders (FWO), project "Multiscale study of reactive gas injection in pyrometallurgical processes," Grant No. G.0433.10N. The authors would like to acknowledge Dr. P. Yan, Dr. Z. Sun, and B. Wu for valuable inputs and inspiring discussions.

${ }^{1}$ G. I. Taylor and P. G. Saffman, "A note on the motion of bubbles in a Hele-Shaw cell and porous medium,” Q. J. Mech. Appl. Math. 12, 265-279 (1959).

2 A. R. Kopf-Sill and G. M. Homsy, "Bubble motion in a Hele-Shaw cell," Phys. Fluids 31, 18-26 (1988).

${ }^{3}$ S. Tanveer, "The effect of surface tension on the shape of a Hele-Shaw cell bubble," Phys. Fluids 29, 3537-3548 (1986)

${ }^{4}$ S. R. K. Maruvada and C. W. Park, "Retarded motion of bubbles in Hele-Shaw cells," Phys. Fluids 8, 3229-3233 (1996).

5 W. Eck and J. Siekmann, "On bubble motion in a Hele-Shaw cell, a possibility to study 2-phase flows under reduced gravity," Ing. Arch. 47, 153-168 (1978)

${ }^{6}$ T. Maxworthy, "Bubble formation motion and interaction in a Hele-Shaw cell," J. Fluid Mech. 173, 95-114 (1986).

${ }^{7}$ R. Collins, "A simple model of the plane gas bubble in a finite liquid," J. Fluid Mech. 22, 763-771 (1965).

${ }^{8}$ F. Riegels, "Zur kritik des Hele-Shaw-versuchs," Z. Angew. Math. Mech. 18, 95-106 (1938).

${ }^{9}$ S. G. Huisman, P. Ern, and V. Roig, "Interaction and coalescence of large bubbles rising in a thin gap," Phys. Rev. E 85, 027302 (2012).

${ }^{10}$ E. Kelley and M. Wu, "Path instabilities of rising air bubbles in a Hele-Shaw cell," Phys. Rev. Lett. 79, 1265-1268 (1997).

${ }^{11}$ J. W. M. Bush, "The anomalous wake accompanying bubbles rising in a thin gap: a mechanically forced Marangoni flow," J. Fluid Mech. 352, 283-303 (1997).

12 J. W. M. Bush and I. Eames, "Fluid displacement by high Reynolds number bubble motion in a thin gap," Int. J. Multiphase Flow 24, 411-430 (1998).

${ }^{13}$ M. Kawaguchi, S. Niga, N. Gou, and K. Miyake, "Buoyancy-driven path instabilities of bubble rising in simple and polymer solutions of Hele-Shaw cell,” J. Phys. Soc. Jpn. 75, 124401 (2006).

${ }^{14}$ H. Kozuka, Y. Ikeda, and M. Kawaguchi, "Path instabilities of bubble rising in polymer solutions of Hele-Shaw cell," J. Phys. Soc. Jpn. 78, 114604 (2009).

${ }^{15}$ M. Yamamoto and M. Kawaguchi, "Differences in path instabilities between a bubble rising in water and in aqueous polymer solution in a Hele-Shaw cell in the transient and steady states," J. Dispersion Sci. Technol. 32, 1445-1451 (2011).

${ }^{16}$ V. Roig, M. Roudet, F. Risso, and A. Billet, "Dynamics of a high-Reynolds-number bubble rising within a thin gap," J. Fluid Mech. 707, 444-466 (2012).

${ }^{17}$ D. Ma, M. Liu, Y. Zu, and C. Tang, "Two-dimensional volume of fluid simulation studies on single bubble formation and dynamics in bubble columns," Chem. Eng. Sci. 72, 61-77 (2012)

${ }^{18}$ W. Boos and A. Thess, "Thermocapillary flow in a Hele-Shaw cell," J. Fluid Mech. 352, 305-330 (1997).

${ }^{19}$ P. Gondret and M. Rabaud, "Shear instability of two-fluid parallel flow in a Hele-Shaw cell," Phys. Fluids 9, 3267-3274 (1997).

${ }^{20}$ A. Tatulchenkov and A. Cebers, "Complex bubble dynamics in a vertical Hele-Shaw cell," Phys. Fluids 17, 107103 (2005).

${ }^{21}$ N. Jarrige, I. Bou malham, J. Martin, N. Rakotomalala, D. Salin, and L. Talon, "Numerical simulations of a buoyant autocatalytic reaction front in tilted Hele-Shaw cells," Phys. Rev. E 81, 066331 (2010).

${ }^{22}$ R. Krishna and J. M. van baten, "Rise characteristics of gas bubbles in a 2D rectangular column: VOF simulation vs experiment," Int. Commun. Heat Mass Transfer 26, 965-974 (1999). 
${ }^{23}$ R. Krishna and J. M. van baten, "Simulating the motion of gas bubbles in a liquid," Nature 398, 208 (1999).

${ }^{24}$ B. Klaasen, F. Verhaeghe, B. Blanpain, and J. Fransaer, "A study of gas bubbles in liquid mercury in a vertical Hele-Shaw cell,” Exp. Fluids 55, 1652 (2014).

${ }^{25}$ J. M. van baten and R. Krishna, "CFD simulations of mass transfer from Taylor bubbles rising in circular capillaries," Chem. Eng. Sci. 59, 2535-2545 (2004).

${ }^{26}$ G. S. Beavers, E. M. Sparrow, and R. A. Magnuson, "Experiments on breakdown of laminar flow in a parallel-plate channel," Int. J. Heat Mass Transfer 13, 809-815 (1970).

${ }^{27}$ I. Lunati and D. Or, "Gravity-driven slug motion in capillary tubes," Phys. Fluids 21, 052003 (2009).

${ }^{28}$ M. van sint annaland, W. Dijkhuizen, N. G. Deen, and J. A. M. Kuipers, "Numerical simulation of behavior of gas bubbles using a 3-D front-tracking method," AIChE J. 52, 99-110 (2006).

${ }^{29}$ G. Tryggvason, R. Scardovelli, and S. Zaleski, Direct Numerical Simulations of Gas-Liquid Multiphase Flows (Cambridge University Press, New York, 2011), p. 105.

${ }^{30}$ J. U. Brackbill, D. B. Kothe, and C. Zemach, “A continuum method for modeling surface tension,” J. Comput. Phys. 100, 335-354 (1991).

${ }^{31}$ M. Seifollahi, E. Shirani, and N. Ashgriz, "An improved method for calculation of interface pressure force in PLIC-VOF methods," Eur. J. Mech. B/Fluids 27, 1-23 (2008).

${ }^{32}$ S. Lin, Y. Chin, C. Wu, J. Lin, and Y. Chen, "A pressure correction-volume of fluid method for simulation of two-phase flows," Int. J. Numer. Methods Fluids 68, 181-195 (2010).

${ }^{33}$ D. L. Youngs, "Time-dependent multi-material flow with large fluid distortion," in Numerical Methods for Fluid Dynamics, edited by K. M. Morton and M. J. Baines (Academic Press, New York, 1982), p. 273.

${ }^{34}$ R. Clift, J. R. Grace, and M. E. Weber, Bubbles, Drops, and Particles (Academic press, New York, 1978), p. 17.

35 J. Zhou, R. J. Adrian, S. Balachandar, and T. M. Kendall, "Mechanisms for generating coherent packets of hairpin vortices in channel flow," J. Fluid Mech. 387, 353-396 (1999).

${ }^{36}$ V. Kolar, "Vortex identification: New requirements and limitations," Int. J. Heat Fluid Flow 28, 638-652 (2007).

${ }^{37}$ B. G. M. Wachem and J. C. Schouten, "Experimental validation of 3-D Lagrangian VOF model: Bubble shape and rise velocity," AIChE J. 48, 2744-2753 (2002).

${ }^{38}$ M. R. Ansari and M. E. Nimvari, "Bubble viscosity effect on internal circulation within the bubble rising due to buoyancy using the level set method," Ann. Nucl. Energy 38, 2770-2778 (2011). 